Research Article

\title{
Research on Walking Gait of Biped Robot Based on a Modified CPG Model
}

\author{
Qiang Lu and Juan Tian \\ College of Information and Engineering, Taishan Medical University, Taian 271016, China \\ Correspondence should be addressed to Qiang Lu; luqiang271016@163.com
}

Received 27 October 2014; Revised 17 December 2014; Accepted 21 December 2014

Academic Editor: Victor Santibáñez

Copyright (C) 2015 Q. Lu and J. Tian. This is an open access article distributed under the Creative Commons Attribution License, which permits unrestricted use, distribution, and reproduction in any medium, provided the original work is properly cited.

The neurophysiological studies of animals locomotion have verified that the fundamental rhythmic movements of animals are generated by the central pattern generator (CPG). Many CPG models have been proposed by scientific researchers. In this paper, a modified CPG model whose output function is $\sin (x)$ is presented. The paper proves that the modified model has stable periodic solution and characteristics of the rhythmic movement using the Lyapunov judgement theorem and the phase diagram. A modified locomotion model is established in which the credit-assignment cerebellar model articulation controller (CA-CMAC) algorithm is used to realize the pattern mapping between the CPG output and the musculoskeletal system. And a seven-link biped robot is employed to simulate cyclic walking gait in order to test the validity of the locomotion model. The main findings include the following. (1) The modified CPG model can generate spontaneous oscillations which correspond to biological signals. (2) The analysis of the modified locomotion model reveals that the CA-CMAC algorithm can be used to realize the pattern mapping between the CPG output and the musculoskeletal system.

\section{Introduction}

Through works of scientific researchers, people have realized that the CPG can generate rhythmic movements. In order to represent the CPG and generate required signals, several nonlinear oscillators that are coupled together have been developed, such as the Hopf Rayleigh, Van del Pol, and Matsuoka oscillators [1-3]. Due to their simplicity and effectiveness, these CPG models have widely been used in robot control and motion simulation of human [4-6].

Humanoid robots have become quite popular and are used as a research tool in many groups worldwide. Among the various motions of a humanoid robot, the most basic and important motion is bipedal walking [7-11]. Chevallereau et al. [7] presented three feedback controllers that achieve an asymptotically stable, periodic, and fast walking gait for a 3D bipedal robot. Shimmyo et al. [8] proposed the biped walking pattern generation by using preview control based on a threemass model. Liu et al. [9] presented biped walking control using a library of optimal trajectories. Wang et al. [10] showed an energy-efficient support vector machine learning control system considering the energy cost of each training sample of biped dynamic in order to realize energy efficient biped walking. Li et al. [11] showed a walking pattern generator based on the control of the center of mass states.

When the CPG model is applied to walking gait, the outputs of the CPG are changed to positions and angles which inspire the musculoskeletal system. Therefore, the outputs of the CPG should correspond to biological signals. But the outputs of Matsuoka model are smoother than biological signals [12]. Taga [13] showed that many parameters which include the body parameters, the parameters in the neural oscillators, the strength of the neural connections, the magnitude of the coefficients in the rhythmic force controller, the strength of the sensory inputs, and the impedance parameters decide the conversion from the CPG to musculoskeletal system in the design of planning gait. Kim et al. [14] showed that the nonparametric estimation based particle swarm optimization is to effectively search the parameters of the CPG. Therefore, they are important works to study the central pattern generator corresponding to biological signals and establish the method to realize the mapping from the CPG to the musculoskeletal system in the fields of robot motor control. The motivations behind this research are to generate 
control signals inspired by biological ones for the robot motions and develop bionic technologies of robots.

This paper is organized as follows. In Section 2, a modified CPG model and a CPG network model are presented. The modified locomotion model and the CA-CMAC algorithm are shown in Section 3. Simulations and results are discussed in Section 4, and the conclusions and future works are made in Section 5.

\section{Modified CPG Model and CPG Network}

2.1. Modified CPG Model. Kimura et al. [15] constructed an oscillator based on Matsuoka's neuron model $[1,2]$. The CPG model is shown in Figure 1. This model consists of two mutually inhibiting neurons that correspond to a flexor neuron and an extensor neuron in animals, respectively. These two neurons alternately induce torque proportional to the inner state in opposite directions. Each neuron is composed of two identical spontaneously firing neurons which remain active even when an animal is at rest and this activity can persist even when synaptic transmission is blocked and is thus endogenously generated. The two neurons are coupled together in such a way that the output of one neuron suppresses the other neuron's activity and vice versa. Together with a certain adaptation or fatigue property of the neurons, the reciprocal inhibition works to produce a stable oscillation [16]. In Figure 1, the filled black circles indicate inhibitory action and the unfilled circles represent excitatory action.

Hence, the CPG model [15-17] can be described by

$$
\begin{aligned}
T_{r} \dot{u}_{i}^{f}+u_{i}^{f} & =-b v_{i}^{f}-w g\left(u_{i}^{e}\right)-\sum w y_{j}+c, \\
T_{a} \dot{v}_{i}^{f}+v_{i}^{f} & =g\left(u_{i}^{f}\right), \\
T_{r} \dot{u}_{i}^{e}+u_{i}^{e} & =-b v_{i}^{e}-w g\left(u_{i}^{f}\right)-\sum w y_{j}+c, \\
T_{a} \dot{v}_{i}^{e}+v_{i}^{e} & =g\left(u_{i}^{e}\right), \\
y_{i} & =g\left(u_{i}^{f}\right)-g\left(u_{i}^{e}\right) .
\end{aligned}
$$

The function $g(\cdot)$ is a piecewise linear function defined by $g(x)=\max (0, x)$, which represents a threshold property of the neurons. These variables $u_{i}^{f}, u_{i}^{e}$, and $y_{i}$ represent the membrane potential which is the difference in electric potential between the interior and the exterior of a biological cell and the firing rate of the neuron, respectively. The two variables $v_{i}^{f}$ and $v_{i}^{e}$ represent the adaptation and fatigue properties that ubiquitously exist in real neurons. The parameter $c$ denotes the tonic input which adapts slowly to a stimulus and continues to produce action potentials over the duration of the stimulus. Parameters $w$ and $b$ represent the strength of mutual and self-inhibition, respectively; parameters $T_{r}$ and $T_{a}$ are the time constants that determine the reaction times of variables $u_{i}^{f}, u_{i}^{e}$ and $v_{i}^{f}, v_{i}^{e}$.

In this model, the entire walking behavior undergoes an adaptation process by causing an oscillation in each joint movement. The movement of a joint is coupled with that

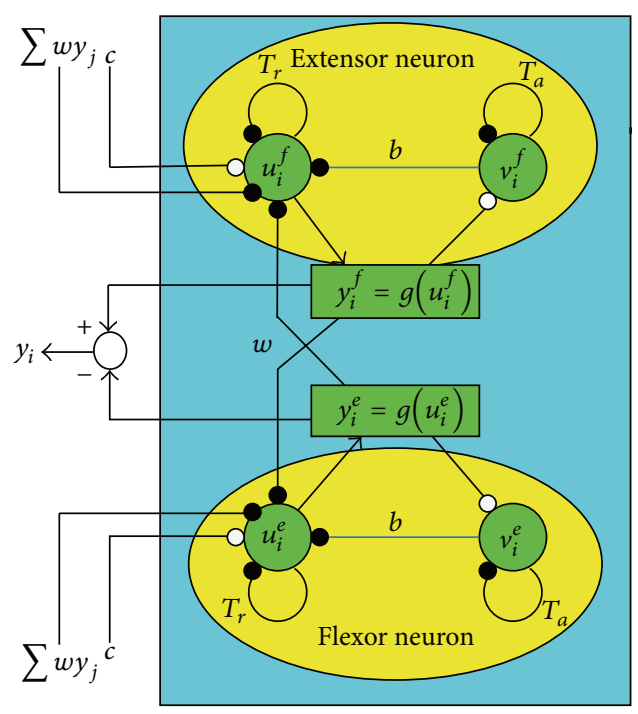

Figure 1: The CPG model.

of other joints. The entire walking behavior converges to a certain stable walking pattern in a harmonious manner through the adaptation process of the oscillator.

In order to analyze this model, we only consider a CPG and $\sum w y_{j}$ in (1) are all omitted. Then (1) is simplified to

$$
\begin{aligned}
& T_{r} \dot{u}_{i}^{f}+u_{i}^{f}=-b v_{i}^{f}-w g\left(u_{i}^{e}\right)+c, \\
& T_{a} \dot{v}_{i}^{f}+v_{i}^{f}=g\left(u_{i}^{f}\right), \\
& T_{r} \dot{u}_{i}^{e}+u_{i}^{e}=-b v_{i}^{e}-w g\left(u_{i}^{f}\right)+c, \\
& T_{a} \dot{v}_{i}^{e}+v_{i}^{e}=g\left(u_{i}^{e}\right) .
\end{aligned}
$$

The signals from nerves are similar to sine [18]. Therefore, $\sin \left(x_{i}\right)$ which can be simulated biological characters is chosen to take the place of $g\left(x_{i}\right)$. Then (3) is obtained:

$$
\begin{aligned}
& \dot{x}_{1}=-5 x_{1}-5 w \sin x_{2}-10 x_{3}, \\
& \dot{x}_{2}=-5 w \sin x_{1}-5 x_{2}-10 x_{4}, \\
& \dot{x}_{3}=0.5 \sin x_{1}-0.5 x_{3}, \\
& \dot{x}_{4}=0.5 \sin x_{2}-0.5 x_{4},
\end{aligned}
$$

where $T_{r}=0.2, T_{a}=2, b=2, c=0, u_{i}^{f}=x_{1}, u_{i}^{e}=x_{2}$, $v_{i}^{f}=x_{3}$, and $v_{i}^{e}=x_{4}$.

Theorem 1. There exists $w$ which makes the equilibrium of system (3) unstable.

Proof. We have the linearization matrix of (3)

$$
\left[\begin{array}{cccc}
-5 & -5 w \cos x_{2} & -10 & 0 \\
-5 w \cos x_{1} & -5 & 0 & -10 \\
0.5 \cos x_{1} & 0 & -0.5 & 0 \\
0 & 0.5 \cos x_{2} & 0 & -0.5
\end{array}\right]
$$


Because the equilibrium is $x_{1}=0, x_{2}=0, x_{3}=0$, and $x_{4}=0$, the above matrix changes to

$$
\left[\begin{array}{cccc}
-5 & -5 w & -10 & 0 \\
-5 w & -5 & 0 & -10 \\
0.5 & 0 & -0.5 & 0 \\
0 & 0.5 & 0 & -0.5
\end{array}\right]
$$

The characteristic equation is

$$
\lambda^{2}+(5.5-5 w) \lambda+7.5-2.5 w=0
$$

or

$$
\lambda^{2}+(5.5+5 w) \lambda+7.5+2.5 w=0 .
$$

The eigenvalues of (6) are

$\lambda_{1,2}=\frac{-(5.5-5 w) \pm \sqrt{(5.5-5 w)^{2}-4(7.5-2.5 w)}}{2}$.

(a) Equation (6) has complex number root for $w \in$ $(0.143,1.743)$. When $w \in(1.1,1.743)$, the equilibrium of nonlinear system (3) is unstable.

(b) Equation (6) has real number root for $w \in(-\infty$, $0.143)$ or $w \in(1.743,+\infty)$. When the sign before radical is positive and $w \in(1.743,+\infty)$, the equilibrium of nonlinear system (3) is unstable. When the sign before radical is negative and $w \in(1.1,3)$, the equilibrium of nonlinear system (3) is unstable.

The eigenvalues of (7) are

$\lambda_{3,4}=\frac{-(5.5+5 w) \pm \sqrt{(5.5+5 w)^{2}-4(7.5+2.5 w)}}{2}$.

(a) Equation (7) has complex number root for $w \epsilon$ $(-1.743,-0.057)$. When $w \in(-1.743,-1.1)$, the equilibrium of system (3) is unstable.

(b) Equation (7) has real number root for $w \in(-\infty$, $-1.743)$ or $w \in(-0.057,+\infty)$. When the sign before radical is positive and $w \in(-\infty,-1.743)$, the equilibrium of system (3) is unstable. When the sign before radical is negative and $w \in(-3,-1.1)$, the equilibrium of nonlinear system (3) is unstable.

According to Lyapunov theorem, the equilibrium of nonlinear system is unstable when there is an eigenvalue whose value or real part is positive. From the above analysis, the equilibrium of nonlinear system (3) is unstable.

Theorem 2. There exists $w$ which makes the system (3) have stable periodic solution.

Proof. According to (3), we can obtain phase diagram of the states $x_{1}, x_{2}, x_{3}$, and $x_{4}$ when initial input value $v_{0}$ is $\left[\begin{array}{llll}0 & 0 & 0 & 0.1\end{array}\right]$ and $w$ is 1.6, as shown in Figure 2.
Based on Poincare-Bendixon's theorem, Zhang et al. [19] derive a lemma.

Lemma 3. The trajectories on the boundary of the region $D_{0}$ are from outside to inside and the ones in the region $D_{0}$ are from inside to outside. If the region $D_{0}$ has no equilibrium except unstable focus, there is stable limit cycle within the region $D_{0}$.

When $w=1.6$, (6) has two complex number roots and their real parts are positive. Therefore, the point $(0,0,0$, 0 ) is an unstable focus. From Figure 2, we can see that one region is composed of the closed trajectories and the directions of these trajectories are from inside to outside. According to Lemma 3, the system (3) has stable limit cycle and stable periodic oscillation.

From the above analysis, there exists $w$ which makes the system (3) have stable periodic solution.

From the proof of Theorems 1 and 2, we can see that this modified CPG model has the characteristics of the rhythmic movement. Therefore, it can be applied to robot movement.

2.2. Network of $C P G$. The CPG oscillators are allocated at each joint and its output is used as joint position commands to the robot. Desired gait is generated by coordinating the movement of multiple oscillators in a self-organizing manner [20]. The CPG network, that is, the arrangement of CPGs to the biped robot, is shown in Figure 3. The topology of the CPG network is annular. These adjacent CPGs are coupled to one another with different values of joint angle.

The six joints' outputs, which are defined as $\phi_{1}, \phi_{2}, \phi_{3}, \phi_{4}$, $\phi_{5}$, and $\phi_{6}$, are shown in Figure 4. Parameters are set as $T_{r}=$ $0.03, T_{a}=0.3, b=1.2$, and $c=2$. Initial input value $v_{1}$ is $\left[\begin{array}{llllllllllllll}0 & 0 & 0 & 0 & 0 & 0 & 0 & 0 & 0 & 0 & 0.1 & 0 & 0.1 & 0.1\end{array}\right]$. In Figure 4, the output of the CPG is similar to biological signal [12].

\section{Human Locomotion Model and CMAC Algorithm}

3.1. Human Locomotion Model. Taga [13] showed the basic structure of the human locomotion. The motor command generation and impedance controller are needed to connect the CPG with the musculoskeletal system. During design process of planning gait, many parameters which include the body parameters, the parameters in the neural oscillators, the strength of the neural connections, the magnitude of the coefficients in the rhythmic force controller, the strength of the sensory inputs, and the impedance parameters are set to obtain stable gait. However, the outputs of coupling CPGs cannot contain spatial information. In this paper, the CMAC algorithm is chosen to replace the motor command generation and impedance controller. The modified human locomotion model is expressed in Figure 5.

3.2. CMAC Algorithm. Albus [21] developed CMAC as a control method based on the principles of the cerebellum's behavior. A CMAC consists of two mappings as shown in Figure 6. 

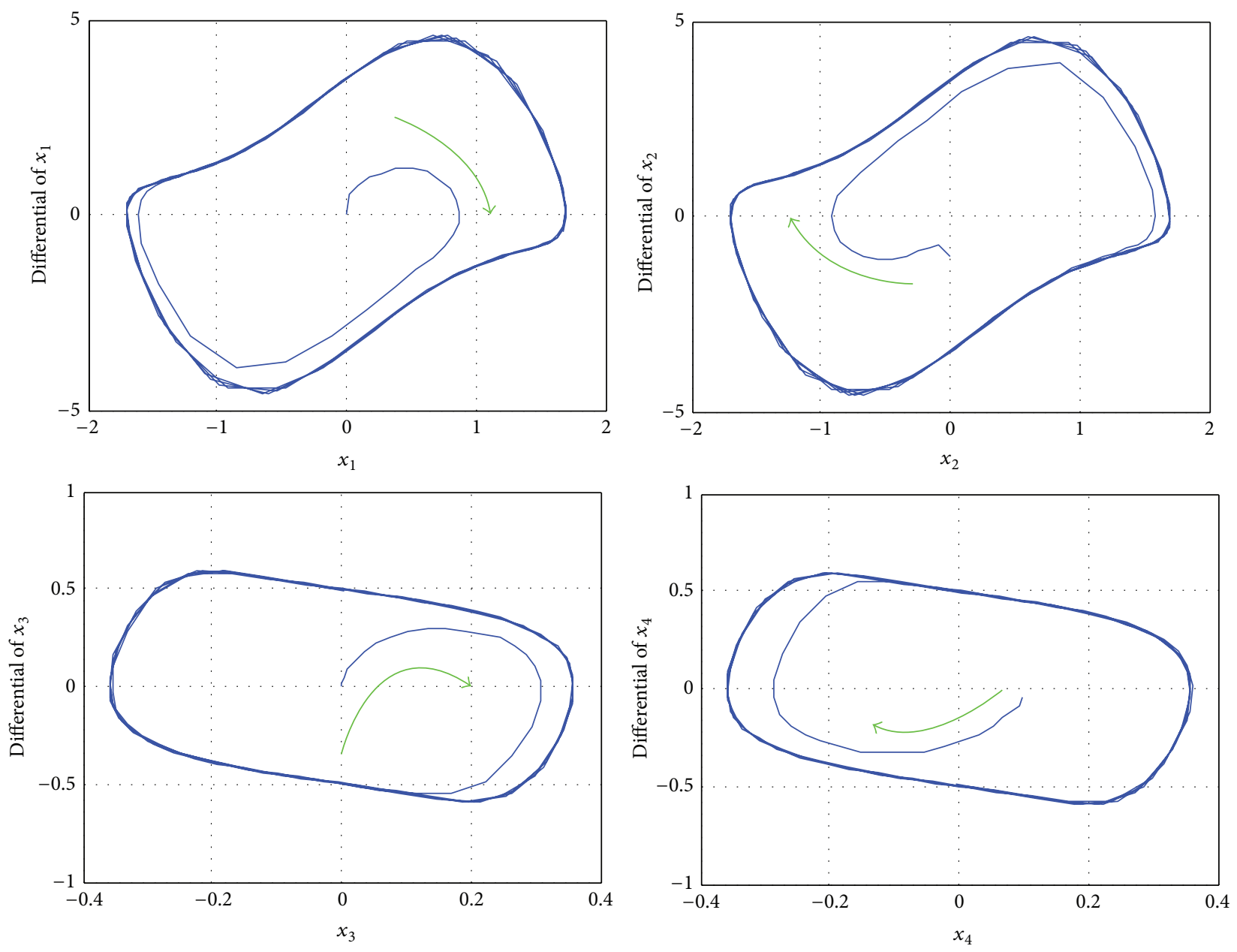

FIGURE 2: Phase diagram with $g(x)=\sin (x)$.

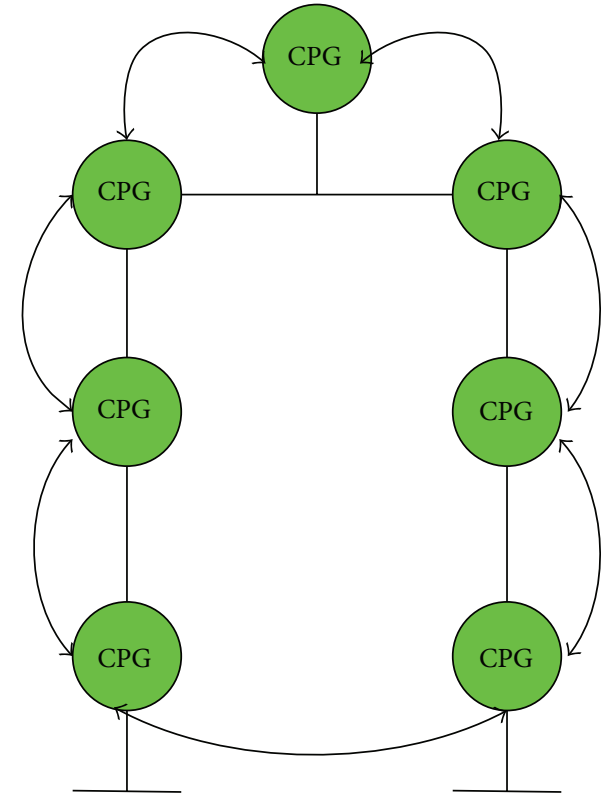

FIgURE 3: Network of CPG.
A fixed mapping $X \rightarrow A$ transforms each $x \in X$ into an $N$ dimensional binary associate vector $a(x)$ in which only $N_{L}$ elements have the value of 1 , where $N_{L}<N$ is referred to as the generalization width. In other words, each $x$ activates precisely $N_{L}$ association cells or geometrically each $x$ is associated with a neighbourhood in which $N_{L}$ association cells are included. The nearby input vectors $x_{i}$ and $x_{j}$ have some overlapping neighborhoods and, therefore, share some common association cells. The degree to which the neighborhoods of $x_{i}$ and $x_{j}$ are overlapping depends on the Hamming distance $H_{i j}$ of $x_{i}$ and $x_{j}$. If $H_{i j}$ is small, the intersection of $x_{i}$ and $x_{j}$ should be large and vice versa. At some values of $H_{i j}$ greater than $N_{L}$, the intersection becomes null, and no local generalization occurs. A second mapping is performed as $A \rightarrow P$. In order to amend the conventional CMAC algorithm, $\mathrm{Su}$ et al. [22] proposed the CA-CMAC whose learning approach is to use the inverse of learned times of the addressed hypercubes as the credibility of the learned values. In comparison to other algorithms, the CA-CMAC has the advantage of very fast learning, and it has the unique property of quickly training certain areas of memory without affecting the whole memory structure, 

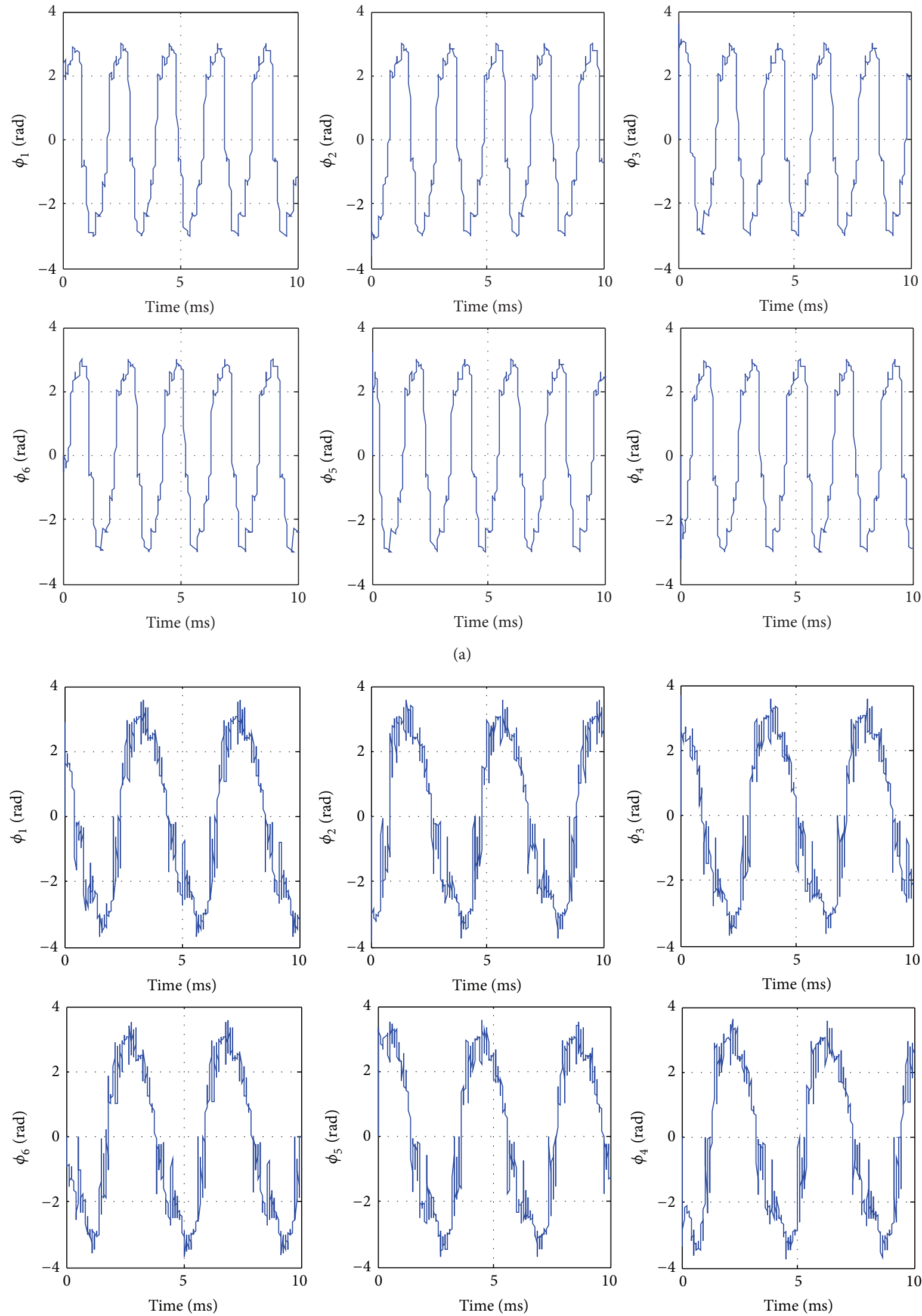

(b)

FIGURE 4: Outputs of the CPG network. (a) $w=2.6$. (b) $w=3.6$. 


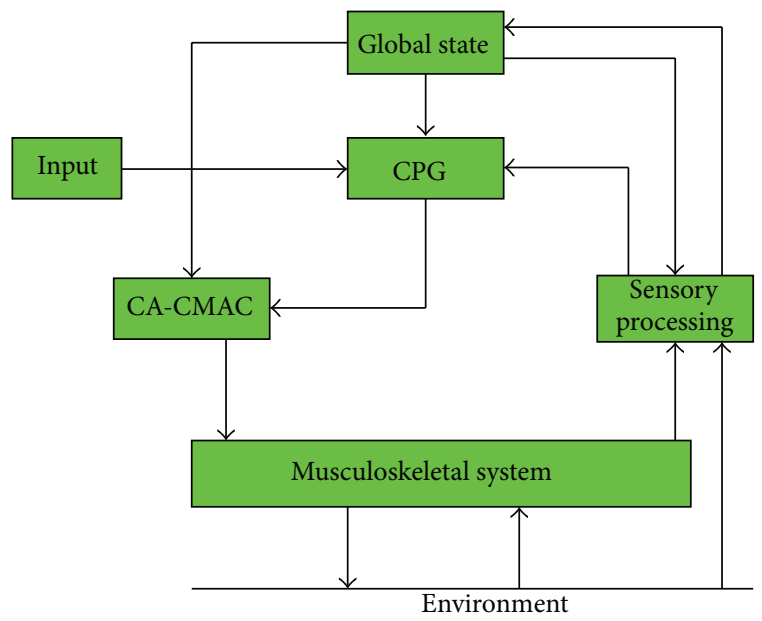

FIGURE 5: Model of human locomotion.

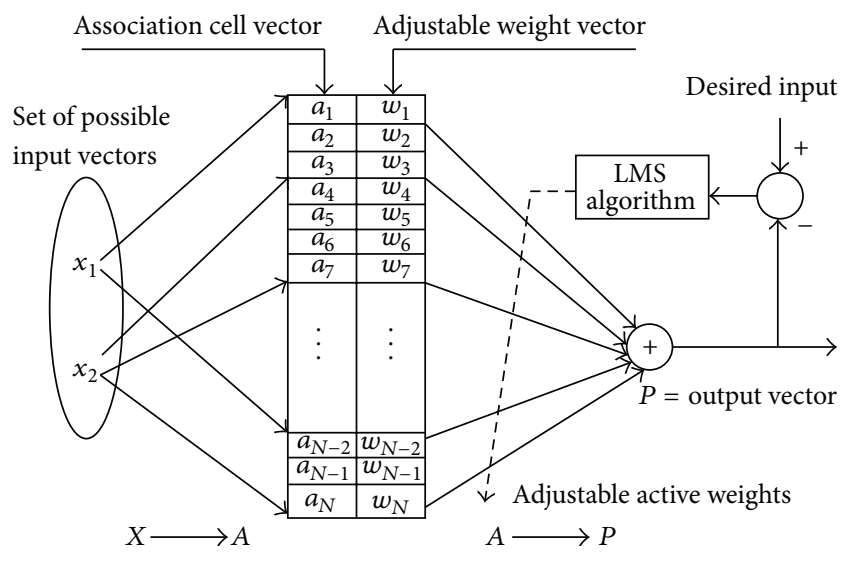

Figure 6: CMAC structure.

which is called generalization. The advantage in training speed is very important in fast gait transition, and the local generalization is particularly suitable for local area features conversion [23-26]. The output of the CA-CMAC is shown in

$$
y_{s}=\sum_{j=1}^{N} C_{s} \omega_{j}
$$

where $N$ is the number of memory elements, $y_{s}$ is the output of state $s$, and $\omega_{j}$ is the weight of the $j$ th memory element. Parameter $C_{s}$ is the flag of whether the $j$ th memory element is activated. If activated, the value is 1 ; otherwise the value is 0 . The update rule of weights is shown in

$$
\begin{aligned}
\omega_{j}^{i}= & \omega_{j}^{i-1}+\alpha C_{s}\left\{\frac{(f(j)+1)^{-1}}{\sum_{l=1}^{m}(f(l)+1)^{-1}}\right\} \\
& \times\left(\overline{y_{s}}-\sum_{j=1}^{N} C_{s} \omega_{j}^{i-1}\right),
\end{aligned}
$$
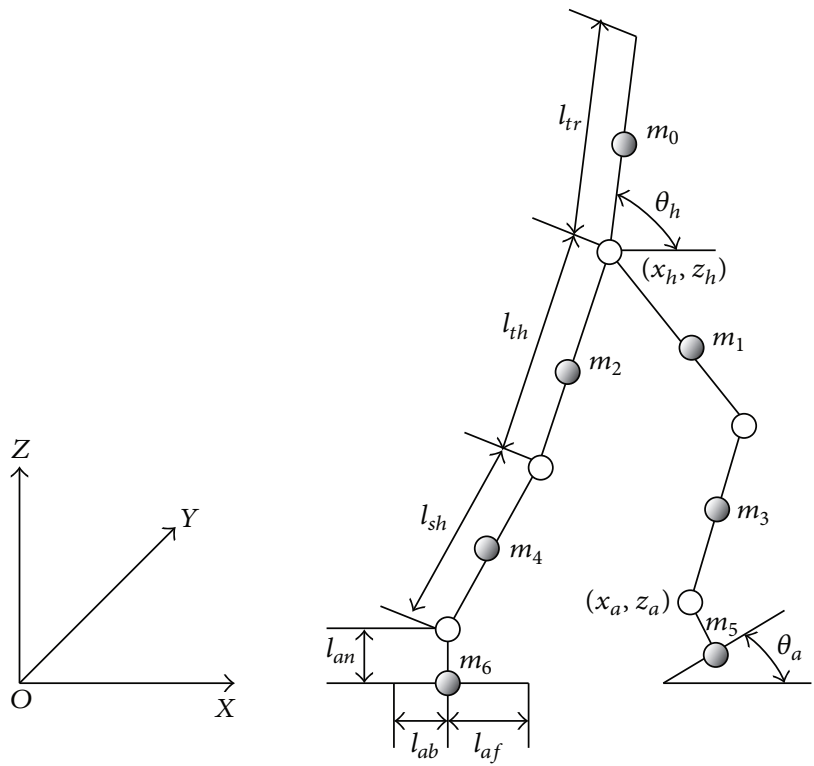

FIGURE 7: Structure of the biped robot.

where $\alpha$ is the learning rate and $f(j)$ is the activated times of the $j$ th memory cell.

\section{Simulations}

The seven-link biped robot [27] is employed to simulate walking gait. The structure is expressed in Figure 7.

In Figure 7, each foot trajectory can be denoted by a vector $X_{a}=\left[x_{a}(t), z_{a}(t), \theta_{a}(t)\right]^{T},\left(x_{a}(t), z_{a}(t)\right)$ is the coordinate of the ankle position, and $\theta_{a}(t)$ denotes the angle of the foot. The hip trajectory can be denoted by a vector $X_{h}=\left[x_{h}(t), z_{h}(t), \theta_{h}(t)\right]^{T},\left(x_{h}(t), z_{h}(t)\right)$ is the coordinate of the hip position, and $\theta_{h}(t)$ denotes the angle of the hip.

Zero moment point (ZMP) is widely employed in the stability control of biped robot locomotion. The ZMP is defined as the point on the ground about which the sum of all the moments of the active forces is equal to zero. To guarantee the stability of the biped robot, the ZMP must be within the support polygon made between the foot and the ground [27]. The robot will fall if the ZMP moves toward the edge of the support polygon. Therefore, the fall of the biped robot can be predicted through monitoring the location of ZMP within the support polygon. The ZMP in $x$-axis direction can be calculated as follows:

$$
x_{\text {zmp }}=\frac{\sum_{i=1}^{n} m_{i}\left(\ddot{y}_{i}+g\right) x_{i}-\sum_{i=1}^{n} m_{i} \ddot{x}_{i} y_{i}}{\sum_{i=1}^{n} m_{i}\left(\ddot{y}_{i}+g\right)},
$$

where $m_{i}$ is the mass of link $i$ and $g$ is the gravitational acceleration. $\left(x_{i}, y_{i}\right)$ is the coordinate of the mass center of link $i$ on an absolute Cartesian coordinate system.

Parameters are set as $l_{t h}=30 \mathrm{~cm}, l_{s h}=30 \mathrm{~cm}, l_{t r}=50 \mathrm{~cm}$, $l_{a n}=10 \mathrm{~cm}, l_{a b}=10 \mathrm{~cm}, l_{a f}=13 \mathrm{~cm}, T_{c}=3 \mathrm{~s}, T_{d}=0.6 \mathrm{~s}$, $q_{b}=0.4 \mathrm{rad}, q_{f}=0.2 \mathrm{rad}, D_{s}=50 \mathrm{~cm}, T_{m}=1.2 \mathrm{~s}$, $L_{a o}=25 \mathrm{~cm}, H_{a o}=16 \mathrm{~cm}, H_{h \text { min }}=73 \mathrm{~cm}, H_{h \max }=74 \mathrm{~cm}$, 

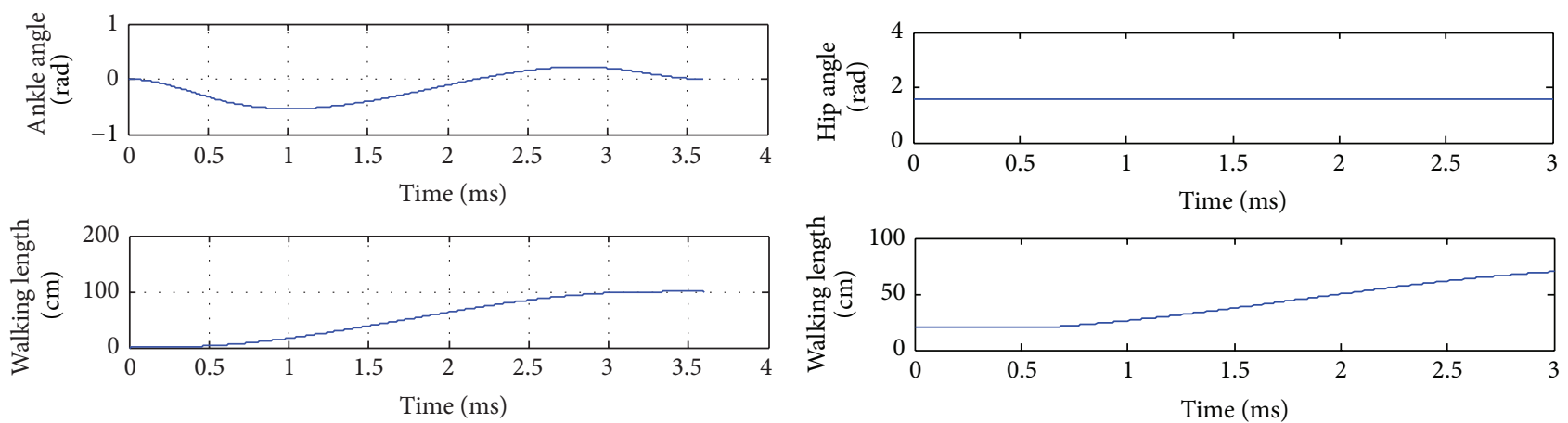

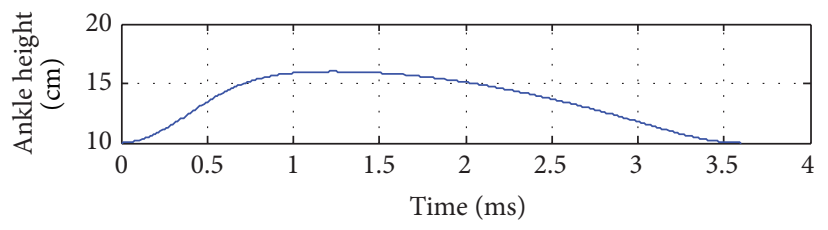

(a)

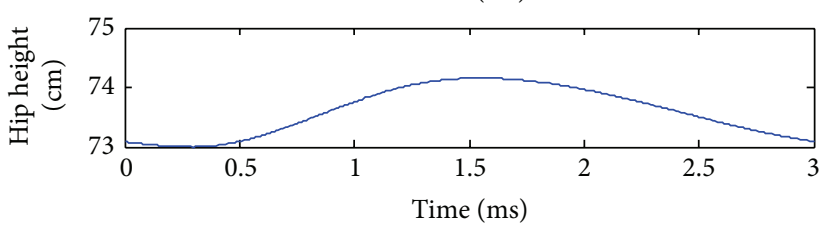

(b)

Figure 8: Curve of the robot's ankle and hip. (a) Curve of the ankle. (b) Curve of the hip.

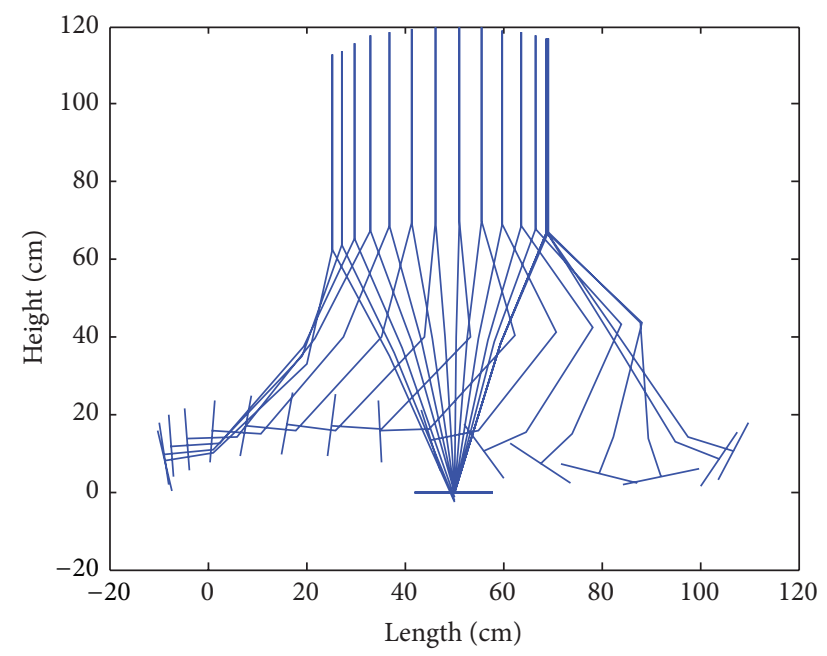

(a)

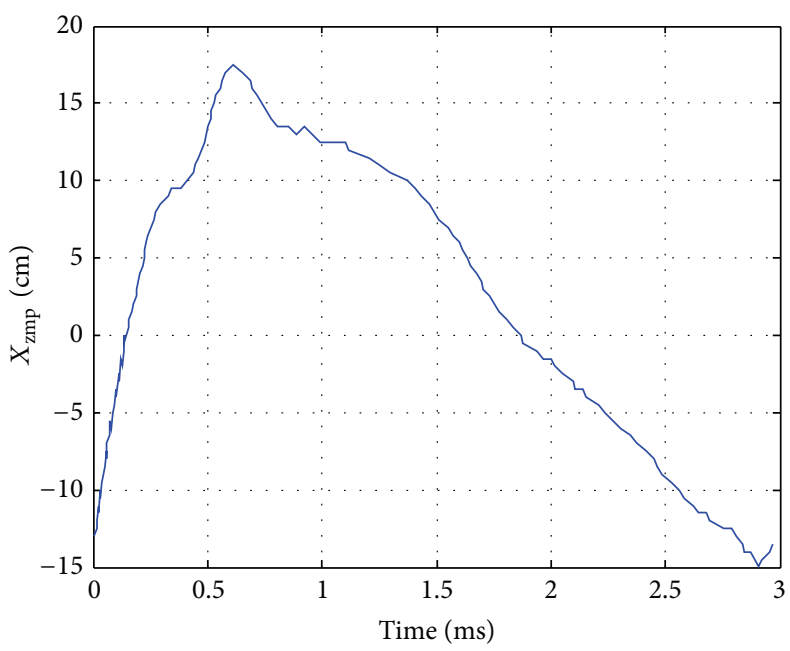

(b)

FIGURE 9: Stick figure of the walking movement and $X_{\text {zmp }}$ based on planning. (a) Stick figure of the walking movement. (b) Diagram of $X_{\text {zmp }}$.

$x_{e d}=20 \mathrm{~cm}$, and $x_{s d}=30 \mathrm{~cm}$ [27]. Then we obtain the curve of the robot's ankle and hip, as shown in Figure 8.

According to planning, the stick figure of the walking movement and the ZMP in the $X$-axis direction are obtained, as shown in Figure 9.

From Figures 8 and 9, we can see both feet leave and land on the ground with the desired angles. The trajectories of motion are smooth and the ZMP trajectory is within the support polygon [27].

In order to recognize the input value of CMAC, a cycle outputs of CPG correspond to cycle planning angles. The input values of CMAC are in two-dimension. The onedimension is the sampling value of CPG, and the other onedimension is the differential value. Six joint angles are needed to be converted in simulation. Hence, six parallel CA-CMACs [28] are chosen for $N=5$ in (10) and $\alpha=0.2$ in (11).

We choose 301 angles, which are obtained from planning gait, as expected values of the CA-CMAC. The 602 twodimension values are obtained by the CPG network. The first 301 two-dimension values are used to train CA-CMAC network. After training, the fitting curve is obtained in Figure 10. In Figure 10, the dotted lines indicate planning angles and the real lines indicate angles which are obtained by six CA-CMACs.

The second 301 two-dimension values are used to test. Then the stick figure of the walking movement is expressed in Figure 11. We also calculate the location of ZMP and $X_{\mathrm{zmp}}$ which is within the support polygon. 

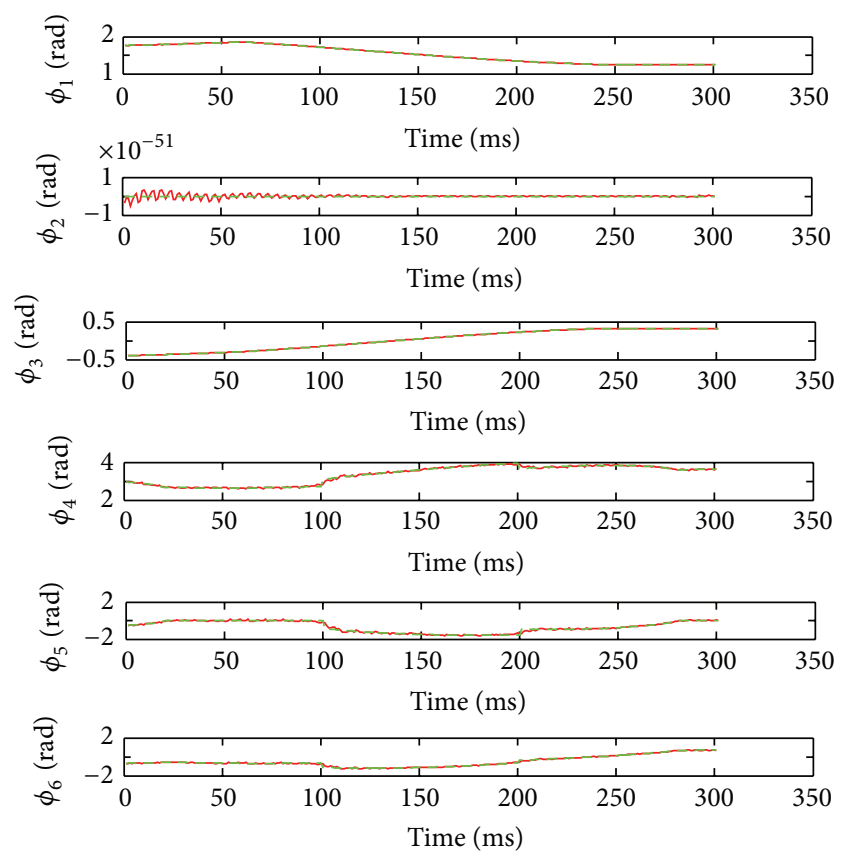

FIGURE 10: Fitting curve between planning angles and output angles based on CA-CMAC.

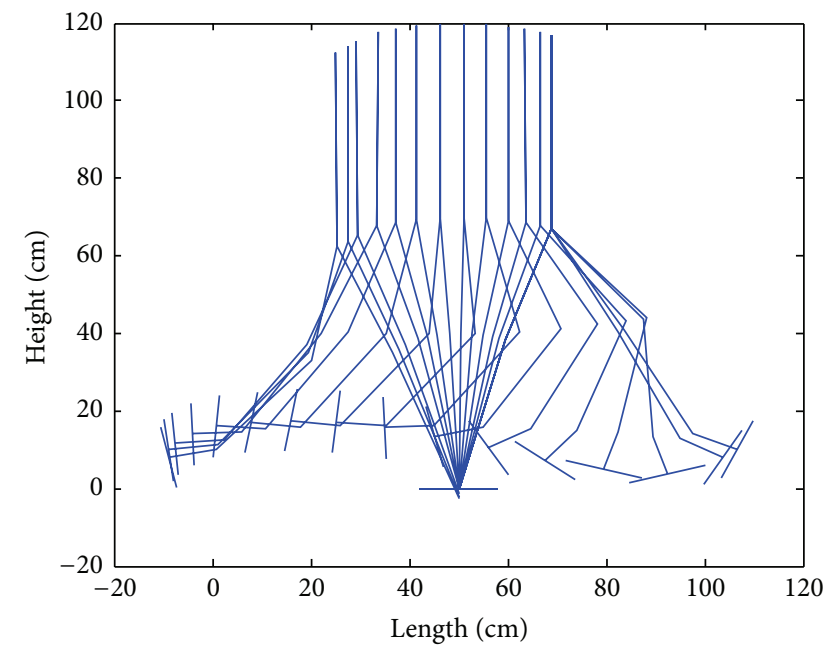

FIGURE 11: Stick figure of the walking movement based on CACMAC.

Comparing Figure 9 with Figure 11, the robot walking gait is also stable with angles which are generated by CA-CMACs.

\section{Conclusion}

The present study is performed on the modified CPG model, the CPG network, and the human locomotion model. The output of CPG can not only be similar to the biological signal, but also inspire musculoskeletal system to obtain natural gait when the CPG parameters are chosen properly. Here, we only discuss the characteristics of CPG model and the locomotion model and their application. The complex dynamic characters of the CPG deserve further investigation.

\section{Conflict of Interests}

The authors declare that there is no conflict of interests regarding the publication of this paper.

\section{References}

[1] K. Matsuoka, "Sustained oscillations generated by mutually inhibiting neurons with adaptation," Biological Cybernetics, vol. 52, no. 6, pp. 367-376, 1985.

[2] K. Matsuoka, "Mechanisms of frequency and pattern control in the neural rhythm generators," Biological Cybernetics, vol. 56, no. 5-6, pp. 345-353, 1987.

[3] J. Yu, M. Tan, J. Chen, and J. Zhang, "A survey on CPG-inspired control models and system implementation," IEEE Transactions on Neural Networks and Learning Systems, vol. 25, no. 3, pp. 441456, 2014.

[4] W. Chen, G. Ren, J. Zhang, and J. Wang, "Smooth transition between different gaits of a hexapod robot via a central pattern generators algorithm," Journal of Intelligent \& Robotic Systems, vol. 67, no. 3-4, pp. 255-270, 2012.

[5] C. Liu, Q. Chen, and D. Wang, "CPG-inspired workspace trajectory generation and adaptive locomotion control for quadruped robots," IEEE Transactions on Systems, Man, and Cybernetics, Part B: Cybernetics, vol. 41, no. 3, pp. 867-880, 2011.

[6] Q. Lu and J. Tian, "Synchronization and stochastic resonance of the small-world neural network based on the CPG," Cognitive Neurodynamics, vol. 8, no. 3, pp. 217-226, 2014.

[7] C. Chevallereau, J. W. Grizzle, and C.-L. Shih, "Asymptotically stable walking of a five-link underactuated 3-D bipedal robot," IEEE Transactions on Robotics, vol. 25, no. 1, pp. 37-50, 2009.

[8] S. Shimmyo, T. Sato, and K. Ohnishi, "Biped walking pattern generation by using preview control based on three-mass model," IEEE Transactions on Industrial Electronics, vol. 60, no. 11, pp. 5137-5147, 2013.

[9] C. Liu, C. G. Atkeson, and J. Su, "Biped walking control using a trajectory library," Robotica, vol. 31, no. 2, pp. 311-322, 2013.

[10] L. Wang, Z. Liu, C. L. P. Chen, Y. Zhang, S. Lee, and X. Chen, "Energy-efficient SVM learning control system for biped walking robots," IEEE Transactions on Neural Networks and Learning Systems, vol. 24, no. 5, pp. 831-837, 2013.

[11] Z. Li, N. G. Tsagarakis, and D. G. Caldwell, "Walking pattern generation for a humanoid robot with compliant joints," Autonomous Robots, vol. 35, no. 1, pp. 1-14, 2013.

[12] M. MacKay-Lyons, "Central pattern generation of locomotion: a review of the evidence," Physical Therapy, vol. 82, no. 1, pp. 69-83, 2002.

[13] G. Taga, "A model of the neuro-musculo-skeletal system for human locomotion I. Emergence of basic gait," Biological Cybernetics, vol. 73, no. 2, pp. 97-111, 1995.

[14] J.-J. Kim, J.-W. Lee, and J.-J. Lee, "Central pattern generator parameter search for a biped walking robot using nonparametric estimation based particle swarm optimization," International Journal of Control, Automation and Systems, vol. 7, no. 3, pp. 447-457, 2009.

[15] H. Kimura, S. Akiyama, and K. Sakurama, "Realization of dynamic walking and running of the quadruped using neural oscillator," Autonomous Robots, vol. 7, no. 3, pp. 247-258, 1999. 
[16] K. Matsuoka, "Analysis of a neural oscillator," Biological Cybernetics, vol. 104, no. 4-5, pp. 297-304, 2011.

[17] Y. Fukuoka, H. Kimura, and A. H. Cohen, "Adaptive dynamic walking of a quadruped robot on irregular terrain based on biological concepts," International Journal of Robotics Research, vol. 22, no. 3-4, pp. 187-203, 2003.

[18] E. Todorov, "Cosine tuning minimizes motor errors," Neural Computation, vol. 14, no. 6, pp. 1233-1260, 2002.

[19] Y.-J. Zhang, Q.-B. Zhu, and E.-G. Tian, "Method of bifurcation analysis of cellular neural network for CPG models," Control Theory and Applications, vol. 23, no. 3, pp. 362-366, 2006.

[20] J. J. Kim and J. J. Lee, "Gait adaptation method of biped robot for various terrains using central pattern generator and learning mechanism," in International Conference on Control, Automation and Systems, vol. 19, pp. 382-387, Seoul, Republic of Korea, 2007.

[21] J. S. Albus, "A new approach to manipulator control: The cerebellar model articulation controller (CMAC)," Journal of Dynamic Systems, Measurement and Control, vol. 97, no. 3, pp. 220-227, 1975.

[22] S.-F. Su, T. Tao, and T.-H. Hung, "Credit assigned CMAC and its application to online learning robust controllers," IEEE Transactions on Systems, Man, and Cybernetics Part B: Cybernetics, vol. 33, no. 2, pp. 202-213, 2003.

[23] C.-S. Lin and C.-T. Chiang, "Learning convergence of CMAC technique," IEEE Transactions on Neural Networks, vol. 8, no. 6, pp. 1281-1292, 1997.

[24] Y.-Q. Wang, Y. Tao, B. Nie, and H.-B. Liu, "Optimal design of motion control for scan tracking measurement: a CMAC approach," Measurement, vol. 46, no. 1, pp. 384-392, 2013.

[25] C. He, L.-X. Xu, and Y.-H. Zhang, "Convergence and generalization ability of CMAC," Control and Decision, vol. 16, no. 5, pp. 523-534, 2001.

[26] X.-M. Lin, T. Mei, M.-Z. Luo, and Y.-F. Song, “The analysis of CMAC generalization," Pattern Recognition and Artificial Intelligence, vol. 19, no. 3, pp. 382-387, 2006.

[27] Q. Huang, K. Yokoi, S. Kajita et al., "Planning walking patterns for a biped robot," IEEE Transactions on Robotics and Automation, vol. 17, no. 3, pp. 280-289, 2001.

[28] Y. Pan, X.-S. Gu, and S.-L. Lu, "Credit-assignment-based parallel ensemble CMAC and its applications in modeling," Control Theory and Applications, vol. 27, no. 2, pp. 211-215, 2010. 


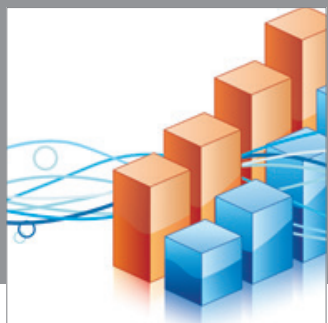

Advances in

Operations Research

mansans

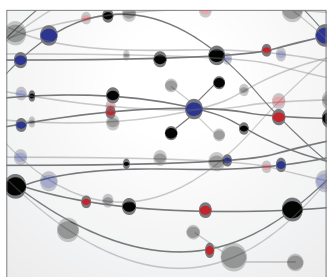

The Scientific World Journal
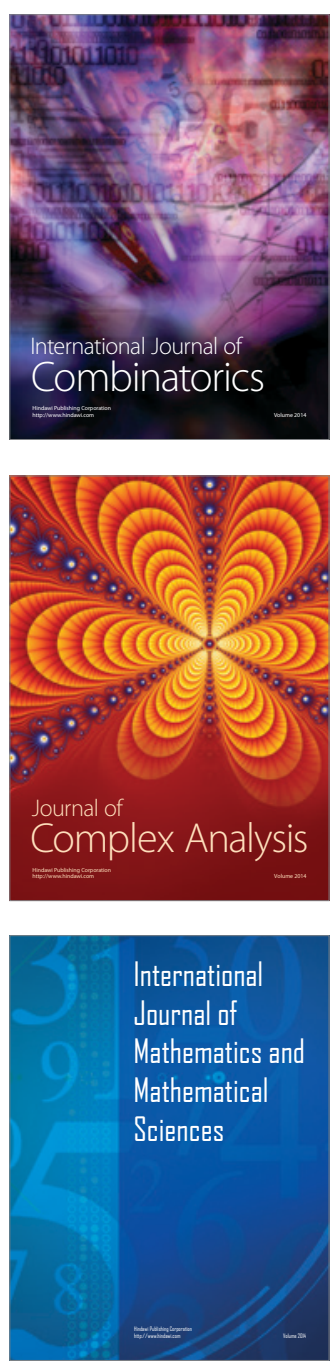
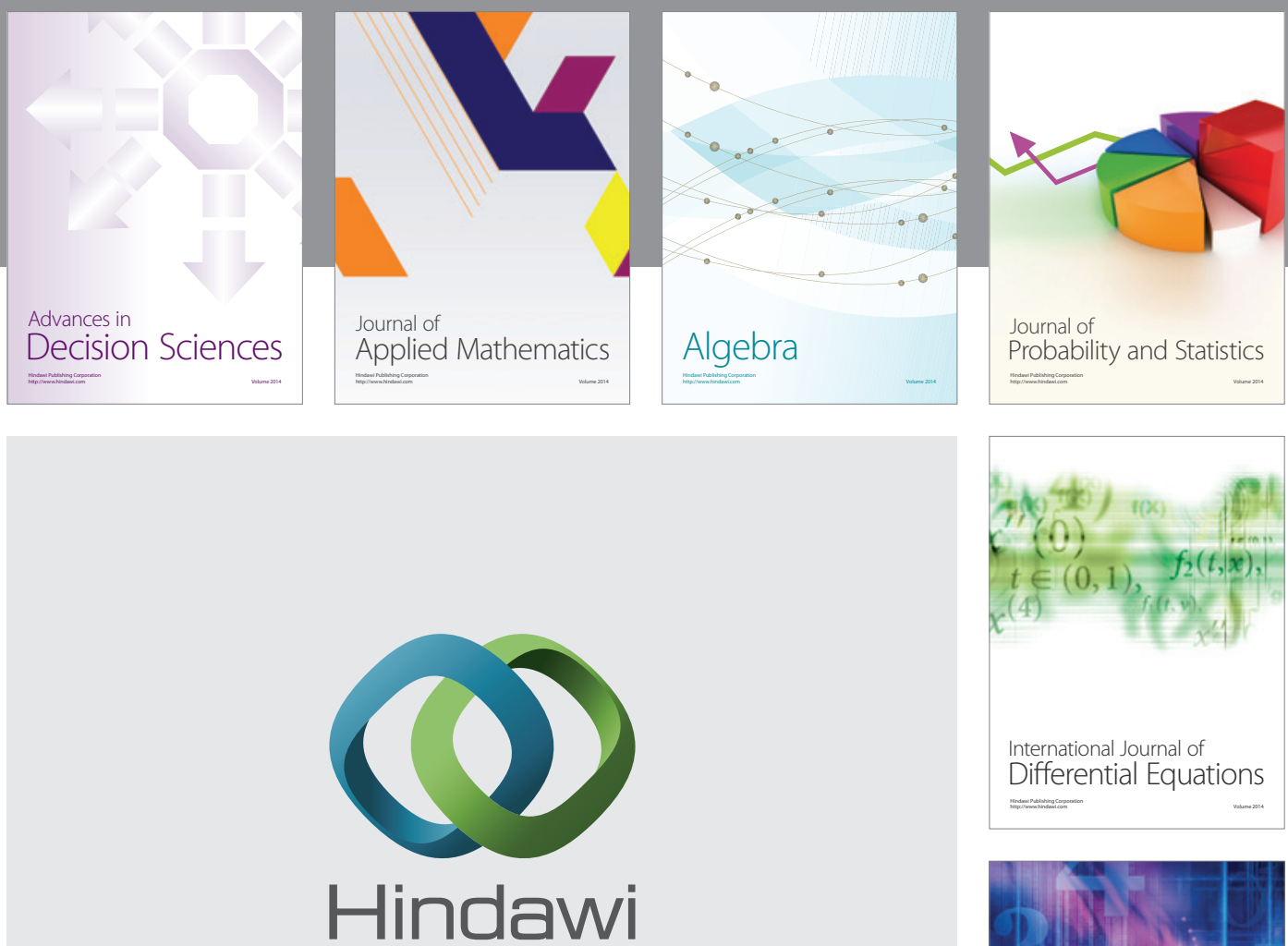

Submit your manuscripts at http://www.hindawi.com
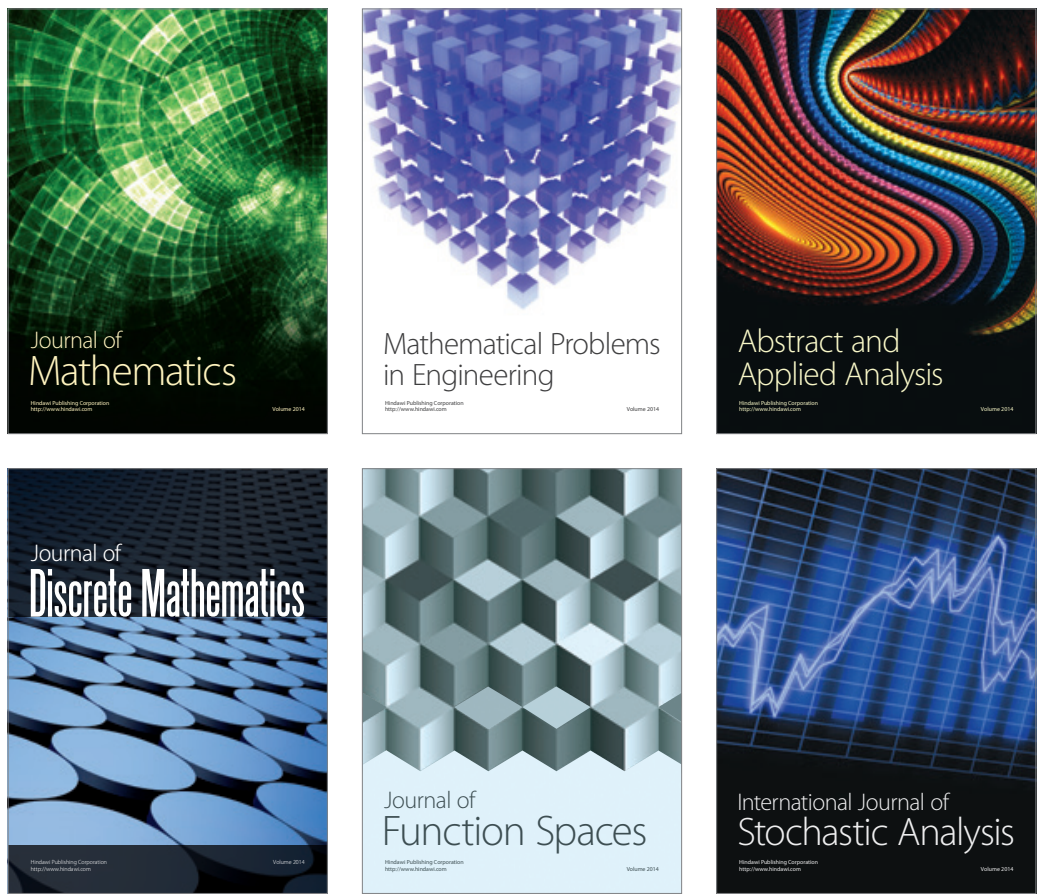

Journal of

Function Spaces

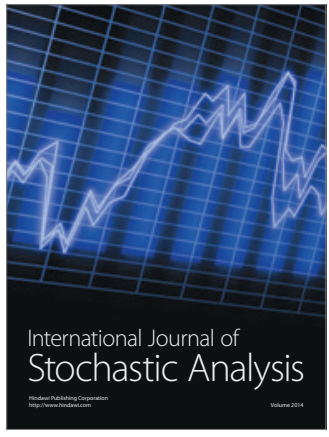

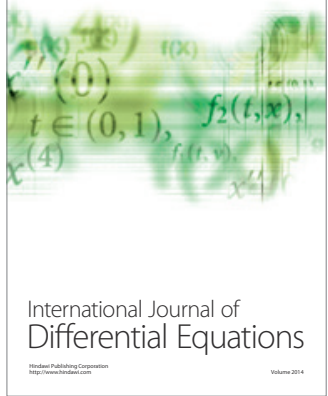
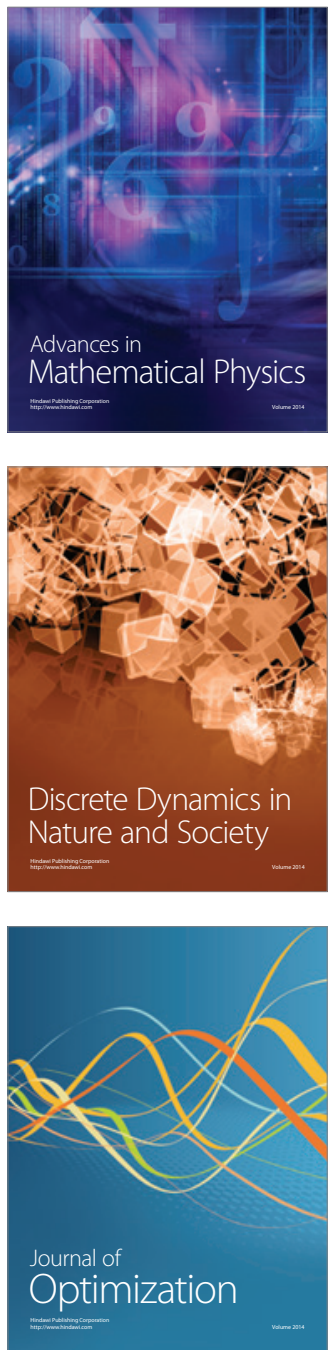